\title{
The neglected part of the microbiome: Prophage TJ1 regulates the bacterial community of the metaorganism Hydra
}

\author{
Authors: Janina Lange; Sebastian Fraune; Thomas C.G. Bosch; Tim Lachnit \\ Authors Affiliation: Zoological Institute, Christian-Albrechts-University Kiel, 24118 Kiel, Germany \\ Corresponding Author: Dr. Tim Lachnit; Zoological Institute; Christian-Albrechts-University Kiel; Am \\ Botanischen Garten 1-9; 24118 Kiel; Germany; phone: +49-431-880-4171; tlachnit@zoologie.uni- \\ kiel.de
}

Keywords: Holobiont | microbiome |host-microbe interaction | virus

\begin{abstract}
Many multicellular organisms are closely associated with a specific bacterial community and therefore considered "metaorganisms". Controlling the bacterial community composition is essential for the stability and function of metaorganisms, but the factors contributing to the maintenance of host specific bacterial colonization are poorly understood. Here we demonstrate that in Hydra the most dominant bacterial colonizer Curvibacter $s p$. is associated with an intact prophage which can be induced by different environmental stressors both in vitro and in vivo. Differences in the induction capacity of Curvibacter phage TJ1 in culture (in vitro) and on Hydra (in vivo) imply that the habitat of the prokaryotic host and/or bacterial frequency dependent factors influence phage inducibility. Moreover, we show that phage TJ1 features a broad host range against other bacterial colonizer and is directly capable to affect bacterial colonization on Hydra. From these results we conclude that prophages are hidden part of the microbiome interfering with bacteria-bacteria interactions and have the potential to influence the composition of host associated bacterial communities.
\end{abstract}




\section{Introduction}

Eukaryotic organisms are living in a close relationship with a complex microbial community, composed of bacteria, fungi, viruses and protists. This close association can be beneficial for both partners and forms a complex unit termed metaorganism or holobiont (Bosch and McFall-Ngai, 2011; Rosenberg et al., 2007). Disturbance or loss of the natural associated bacterial community can facilitate the invasion of pathogens and lead to reduced host fitness (Van Rensburg et al., 2015; Bates et al., 2018; Fraune et al., 2014).

Host genetics and innate immunity play an important role in establishing and maintaining the microbial composition of metaorganisms. For some animal species including Hydra it has been shown that the eukaryotic host actively selects and shapes its specific bacterial community (Franzenburg et al., 2013a; Goodrich et al., 2014; Franzenburg et al., 2012; Pietschke et al., 2017). In Hydra, bacteria-bacteria interactions are an important component contributing to the fitness of the metaorganism (Fraune et al., 2014). The most abundant bacterial colonizer of Hydra vulgaris (AEP) is the proteobacteria Curvibacter sp.. Interestingly, Curvibacter can protect the animal host from fungal infection if one of the other main colonizers such as Duganella sp. (11\%), Undibacterium sp. (2.1\%) or Pelomonas sp. $(0.2 \%)$ is also present (Fraune et al., 2014). Co-occurrence of these bacteria is thus essential to provide this beneficial antifungal host defense. Li et al. (2015) investigated the interaction between the two most abundant bacterial colonizers of Hydra, Curvibacter $\mathrm{sp}$. and Duganella sp., in vitro and observed that in mono-culture Duganella sp. features higher growth rate than Curvibacter sp.. In contrast to the expectation that Duganella $s p$. would outcompete Curvibacter sp. in co-culture experiments, a frequency dependent suppression of Duganella sp. was detected (Li et al., 2015). The observation of a frequency dependent growth rate indicates that the interactions among bacteria in co-culture are beyond a simple case of direct competition and it has been predicted by modelling that this interaction is mediated by a temperate phage integrated in the genome of Curvibacter sp. (prophage) (Li et al., 2017). The fresh water polyp Hydra is not only associated with a host specific bacterial community (Franzenburg et al., 2013b) but also features a host specific viral community of which more than $50 \%$ are bacteriophages (Grasis et al., 2014). Due to the fact that phages are often obligate killers to their host cells but also to other bacteria, they have a strong selective effect on bacterial populations and are hypothesized to shape whole bacterial communities (Bohannan and Lenski, 2000; Gómez and Buckling, 2011; Suttle, 2007; Koskella and Meaden, 2013). While lytic phages infect bacteria, get multiplied and kill their bacterial host (Lenski, 1988), temperate phages can undergo lysogenic conversion, where the phage genome is replicated along with the genome of its host and is transferred vertically (De Paepe et al., 2014). By this they can increase the fitness of its bacterial host by altering the bacterial geno- and phenotype (Oakey and Owens, 2000). During stress for the bacterial host cell, temperate phages can switch from lysogenic to lytic cycle (prophage induction) (Ranquet et al., 2005; Nanda et al., 2015). Free phages are then released to the environment and are able to cross-infect and kill other bacteria (Livny and Friedman, 2004; Refardt, 2012). In this manner excised prophages may function as weapons in competition with other (susceptible) bacteria (Bossi et al., 2003; Burns et al., 2015). In a previous study we could demonstrate that the prophage of Curvibacter sp. is inducible and can lytically infect the second most abundant colonizer of Hydra Duganella sp. in vitro. In the present study we further 
investigate the function of Curvibacter phage (TJ1) in the host context and hypothesized that phage TJ1 interferes with bacteria-bacteria interactions and has the potential to influence the composition of host associated bacterial community. To test this hypothesis, we investigated the presence and induction capacity of the prophage associated with Curvibacter sp. and its ability to cross-infect and downregulate other Hydra associated bacteria in culture (in vitro) and on Hydra (in vivo).

Our results demonstrate that Curvibacter phage (TJ1) can be induced in culture (in vitro) and on Hydra (in vivo). In association with Hydra, Curvibacter sp. was more susceptible for phage induction at reduced water temperature, suggesting an impact of the host environment on phage bacteria interactions. Finally, we could demonstrate that phage TJ1 has a broad host range against other bacterial colonizers and has the potential to regulate bacterial colonization on Hydra.

\section{Material and Methods}

Identification and induction of Curvibacter prophage. First, we screened the bacterial genome of Curvibacter sp. (strain AEP1.3; GenBank:CP015698.1) for the presence of prophage signatures using the online software PHASTER (Phage Search Tool Enhanced Release) (Arndt et al., 2016). Second, we used Mitomycin C assay to test the induction capacity of the phage. Therefore, Curvibacter sp. was grown in 3\% (w/v) R2A broth media (Sigma-Aldrich) under shaking conditions at 250 $\mathrm{rpm}$ at $18^{\circ} \mathrm{C}$. Exponentially growing bacterial overnight cultures were inoculated with $0.05 \mu \mathrm{g} / \mathrm{ml}$ Mitomycin C to induce phage replication (Sekiguchi and Takagi, 1960). After an incubation time of $16 \mathrm{~h}$ bacterial cells were removed by two consecutive lowspeed-centrifugation steps at 4,266 x $\mathrm{g}$ in a ThermoScientific Heraeus Multifuge 3SR at $4^{\circ} \mathrm{C}$ for $30 \mathrm{~min}$. The supernatant was filtered $0.2 \mu \mathrm{m}$ and phage particles were pelleted by ultracentrifugation at $25,000 \mathrm{rpm}(72,700 \mathrm{x} \mathrm{g})$ in a Beckman 45 Ti rotor at $4^{\circ} \mathrm{C}$ for $2 \mathrm{~h}$. The pellet was re-suspended in $3 \mathrm{ml}$ SM-Buffer $(50 \mathrm{mM}$ Tris; $100 \mathrm{mM}$ $\mathrm{NaCl} ; 8 \mathrm{mM} \mathrm{MgSO} 4$; $\mathrm{pH} 7.5)$. Re-suspended phages were layered onto a pre-formed Cesium chloride gradient consisting of the densities $\left(2 \mathrm{ml}: 1.7 ; 1.5 ; 1.3 ; 1.2 \mathrm{~g} / \mathrm{cm}^{3}\right.$ and $\left.1 \mathrm{ml} 1.1 \mathrm{~g} / \mathrm{cm}^{3}\right)$ in SM-buffer and centrifuged at $28.000 \mathrm{rpm}(135,000 \mathrm{x} \mathrm{g})$ in a Beckman SW 41 rotor at $4^{\circ} \mathrm{C}$ for $2 \mathrm{~h}$. The band containing phages was removed by syringe, diluted 1:3 in SM-Buffer and pelleted by centrifugation at $22000 \mathrm{rpm}(83,000$ $\mathrm{x} \mathrm{g}$ ) in a Beckman SW 41 rotor at $4^{\circ} \mathrm{C}$ for $2 \mathrm{~h}$. Phages were re-suspended in $200 \mu \mathrm{l}$ SM-Buffer and stored at $-80^{\circ} \mathrm{C}$ until DNA extraction. Sub-samples of phages $(5 \mu \mathrm{l})$ were further characterized morphologically by negative staining in $2 \%(\mathrm{w} / \mathrm{v})$ aqueous uranyl acetate and visualized by transmission electron microscopy (TEM) using a Technai Bio TWIN at $80 \mathrm{kV}$ and a magnification of 40 000-100 000.

Phage DNA extraction and Sequencing. Phage DNA was extracted from twohundred microliters of purified phages according to the protocol developed by Thurber and colleagues (Thurber et al., 2009) with minor modifications. In brief, 22 $\mu$ l $2 \mathrm{M}$ Tris-HCL (pH 8.5)/0.2 M EDTA, $10 \mu \mathrm{l} 0.5 \mathrm{M}$ EDTA and $268 \mu \mathrm{l}$ formamid were added to the samples and incubated for $30 \mathrm{~min}$ at room temperature. DNA was precipitated by adding two volumes of ethanol and incubation at $-20^{\circ} \mathrm{C}$ overnight. DNA was pelleted by centrifugation at $13,000 \times \mathrm{g}$ at $4^{\circ} \mathrm{C}$ for $20 \mathrm{~min}$. The pellet was washed with $70 \%$ (v/v) ethanol. $100 \mu \mathrm{l}$ SDS extraction buffer (1\% SDS; $100 \mathrm{mM}$ Tris; $20 \mathrm{mM}$ EDTA; $\mathrm{pH} 7.5), 1 \%$ 2-mercaptoethanol and proteinase $\mathrm{K}(0.5 \mathrm{mg} / \mathrm{ml})$ were added to the pellet and incubated for $30 \mathrm{~min}$ at $36^{\circ} \mathrm{C}$ and $15 \mathrm{~min}$ at $56^{\circ} \mathrm{C}$. Twice the amount of DNA-extraction buffer (100 mM Tris, pH 8.0; $1.4 \mathrm{M} \mathrm{NaCl} ; 20 \mathrm{mM}$ EDTA; $1 \%$ 2-mercaptoethanol; $2 \%(w: v)$ CTAB) were added followed by an incubation step at $65^{\circ} \mathrm{C}$ for $10 \mathrm{~min}$. An equal volume of chloroform:isoamyl alcohol (24:1) was added 
to the warm solution, mixed thoroughly and centrifuged at $13,000 \times g$ at room temperature for 5 min. Supernatant was transferred into a new tube and DNA was precipitated by the addition of 0.7 volume of isopropanol. After an incubation at $-20^{\circ} \mathrm{C}$ for $2 \mathrm{~h}$ DNA was pelleted by centrifugation at $13,000 \times \mathrm{g}$ at $4^{\circ} \mathrm{C}$ for $20 \mathrm{~min}$. DNA was washed with $70 \%(\mathrm{v} / \mathrm{v})$ ethanol and dissolved in $50 \mu \mathrm{l}$ water. Nextera XT kit (Illumina) was used for library preparation and $2 \times 150$ bp paired-end sequencing was conducted on a MiSeq platform (Illumina) at Centre for molecular biology in Kiel. Trimmomatic V.0.36 (Bolger et al., 2014) was used for sequence adaptor removal and read trimming. Trimmed and quality controlled reads were finally assembled using SPAdes V.3.1.11 (Bankevich et al., 2012). The assembled Curvibacter phage TJ1 genome is publically available under the GenBank accession number MH766655 in the NCBI database.

Phage annotation and comparison. Phage TJ1 was annotated using the genome annotation service RAST (Rapid Annotation using Subsystem Technology). The phage capsid protein was compared with different phages by Psi-Blast. The most closely related was Burkholderia phage KS10 (NCBI: Reference Sequence: NC_011216.1) by SEED Viewer (Wattam et al., 2017). Gene organization and sequence similarity between Curvibacter phage TJ1 and Burkholderia phage KS10 was graphically illustrated by SEED Viewer centered on the phage capsid protein (Wattam et al., 2017).

In a next step we controlled RAST predicted ORFs by GeneMark (Borodovsky and Mclninch, 1993) and conducted similarity searches by BLASTP (Altschul et al., 1997), SWISS-PROT (Bairoch and Apweiler, 2000), UniProt (2019) and Pfam databases (El-Gebali et al., 2019). Based on BLASTP results we selectively downloaded bacterial genomes from the National Centre for Biotechnology Information (NCBI) and screened them for the presence of prophages by PHASTER (Phage Search Tool Enhanced Release) (Arndt et al., 2016). Prophage sequences were extracted and submitted together with the sequence of Burkholderia phage KS10 to VICTOR (Meier-Kolthoff and Göker, 2017) for phylogenetic analysis using the GENOME-BLAST Distance Phylogeny method (GBDP) (Meier-Kolthoff and Göker, 2017).

Phage quantification. Phages were quantified by qPCR (Imamovic et al., 2010; Refardt, 2012). In order to take into account that phage sequences derive from either induced phages or from bacterial cells carrying integrated prophages in their genome we quantified both phage TJ1 and bacteria via qPCR. We calculated the ratio between phage and bacteria to exclude the effect of differences in growth or polyp size. For the quantification of phage TJ1 we designed a specific set of primers targeting the phage tail gene (F: 5'-GCTTTGACCTGTCGTTCATCC-3' and R: 5'CGGGTTTGTTGGATAGGTCGT-3'). For bacteria quantification we used either primer specific for the recA gene of Curvibacter sp. (strain AEP1.3) (F: 5'TTCGGCAAGGGCACCATC-3' and R: 5'-ACGACTCCGGGCCATAGA-3') or Eubacterium Primer (F: 5'-CCTACGGGAGGCAGCAG-3' and R: 5'ATTACCGCGGCTGCTGGC-3') for quantification of the other bacterial colonizer (cross-infection experiment in vivo). Tests of primer sets on DNA extracts of germ free control polyps were negative. QPCR reactions were performed in a $25 \mu$ volume with $12.5 \mu \mathrm{l}$ GoTaq qPCR MasterMix (Promega), $10 \mathrm{pmol} / \mu \mathrm{l}$ forward and reverse primers and $10 \mathrm{ng} / \mu \mathrm{l}$ template DNA. Cycler (Applied Biosystems 7300 Real Time PCR System) conditions were as follows: $95^{\circ} \mathrm{C}$ for $2 \mathrm{~min}, 40$ cycles of $95^{\circ} \mathrm{C}$ for $15 \mathrm{~s}$, $59^{\circ} \mathrm{C}$ for $30 \mathrm{~s}, 60^{\circ} \mathrm{C}$ for $35 \mathrm{~s}$, followed by a dissociation step. At the end of each cycle 
at $60^{\circ} \mathrm{C}$ the fluorescence was measured. Sequence data are deposited at Sequence Read Archive

(BioSample accessions: SAMN11334657-SAMN11334682).

Environmental stress induction of phage TJ1 in vitro. Bacterial log phase cultures were grown in R2A media until they reached an optical density (OD600) of 0.26 . Subsequently $6 \mathrm{ml}$ bacterial cultures were exposed separately and with replication $(n=5)$ to one of the following conditions, while the rest of the environmental conditions remain normal: altered temperature $\left(23^{\circ} \mathrm{C}, 12^{\circ} \mathrm{C}\right)$, elevated $\mathrm{pH}(9.5,8.5)$, higher nutrition $(4 \times \mathrm{R} 2 \mathrm{~A}$ medium $(12 \mathrm{~g} / \mathrm{l}))$ or normal conditions $\left(18^{\circ} \mathrm{C}\right.$, $\mathrm{pH} 7$ ) serving as control. After an incubation time of $16 \mathrm{~h}$, DNA was extracted using the DNA blood and tissue kit (Qiagen) followed by phage and Curvibacter sp. quantification by qPCR (see above).

Recolonization of Hydra. Experiments were carried out using Hydra vulgaris (AEP) (Hemmrich et al., 2007). Prior to recolonization animals were cultured under constant laboratory conditions including Hydra culture medium $(0.28 \mathrm{mM} \mathrm{CaCl} 2,0.33 \mathrm{mM}$ $\mathrm{MgSO}_{4}, 0.5 \mathrm{mM} \mathrm{NaHCO}_{3}$ and $0.08 \mathrm{mM} \mathrm{KCO}_{3}$ ), food (first instar larvae of Artemia salina, fed four times per week) and constant water temperature at $18^{\circ} \mathrm{C}$, according to standard procedures (Bosch et al., 1988). Germfree Hydra were generated by exposing animals to an antibiotic cocktail containing $50 \mu \mathrm{g} / \mathrm{ml}$ of Ampicillin, Rifampicin, Streptomycin, Spectinomycin and Neomycin as previously described (Franzenburg et al., 2012). Antibiotic solutions were exchanged every second day. After 2 weeks of antibiotic treatment animals were transferred to antibiotic free sterile Hydra culture medium for 3 days. Sterility was controlled with previous established methods (Franzenburg et al., 2013b). $5000 \mathrm{CFU} / \mathrm{ml}$ of the corresponding bacterial strain was added to the surrounding water $(50 \mathrm{ml})$ of germfree polyps and incubated for one day. Afterwards polyps were washed in sterile Hydra medium in order to remove unattached bacteria from the surrounding medium and transferred into new container with fresh, sterile Hydra medium and used for subsequent experiments. Depending on the bacterial strain initial abundance of monocolonised Hydra was roughly $15-400$ CFU per polyp.

Environmental stress induction of phage TJ1 in vivo. Curvibacter $\mathrm{sp}$. monocolonized Hydra polyps (see above) were transferred to $1.5 \mathrm{ml}$ tubes with 200 $\mu \mathrm{l} \mathrm{Hydra}$ culture medium. After 3 days of settlement they were exposed separately and with replication $(n=5)$ to one of the following conditions, while the rest of the environmental conditions remain normal: altered temperature $\left(23^{\circ} \mathrm{C}, 12^{\circ} \mathrm{C}\right)$, higher $\mathrm{pH}(9.5,8.5)$ and elevated nutrition $(10 \% \mathrm{R} 2 \mathrm{~A}$ diluted in sterile Hydra culture medium) or normal conditions $\left(18^{\circ} \mathrm{C}, \mathrm{pH} 7\right)$ serving as control. After $16 \mathrm{~h}$ incubation time DNA was extracted from the polyp including surrounding water. Phages and Curvibacter were quantified by qPCR.

Cross-infection and host range assay in vitro. Cross-infectivity of phage TJ1 was tested by spot assays in double agar layer (Adams, 1959) against our bacterial culture collection consisting of diverse bacterial strains isolated from different Hydra species (see Table 1). Exponentially growing bacterial strains were mixed into $5 \mathrm{ml}$ preheated top-agar (R2A medium, $5 \mathrm{mM} \mathrm{CaCl}_{2}, 5 \mathrm{mM} \mathrm{MgCl}, 0.4 \%$ agarose) and poured onto R2A agar plates (R2A medium, 1.5\% agar). Plates were dried for at least $30 \mathrm{~min}$ and $10 \mu \mathrm{l}$ of purified phages and $10 \mu \mathrm{l}$ sterile Hydra culture medium as control were spotted onto each bacterial lawn $(n=3)$. Plaque formation was checked 
after incubation at $18^{\circ} \mathrm{C}$ for $12-24 \mathrm{~h}$. Purity of phages was controlled for the presence of potential bacterial contaminations by plating out the phage suspension on R2A agar plates.

Cross-infection assay in vivo. This experiment was conducted with a subset of bacterial strains specific for the colonization of Hydra vulgaris (AEP). Germfree Hydra vulgaris (AEP) polyps were monocolonized with Curvibacter sp., Duganella sp., Undibacterium sp., Acidovorax sp. or Pelomonas sp. (see above) in log-phase.After 3 days of settlement, the recolonized polyps were transferred separately to $1.5 \mathrm{ml}$ tubes, containing $200 \mu \mathrm{l}$ sterile Hydra culture medium. Phage TJ1 was purified from $100 \mathrm{ml}$ culture and resuspended in sterile Hydra culture medium andwere then added to 5 monocolonized polyps at a concentration of $1 \times 10^{4} \mathrm{PFU} / \mathrm{ml}$. As control served 5 monocolonized polyps that received an equal volume of pure sterile Hydra culture medium. After $0 \mathrm{~h}, 24 \mathrm{~h}$ and $72 \mathrm{~h}$ post infection the amount of colonizing bacteria and the amount of phages were quantified. For bacterial quantification polyps were homogenized separately, diluted and plated out onto R2A agar plates $(n=5)$ and incubated at $18^{\circ} \mathrm{C}$. CFU per polyp were counted after 2-4 days of incubation. For the quantification of phages that were released to the surrounding water, DNA was extracted from the polyps and their surrounding sterile Hydra culture medium, using the DNA Blood and Tissue Kit (Qiagen). Phages and bacteria were quantified by qPCR (see above).

Phage induction by different bacterial colonizers in vivo. In order to test if phage TJ1 is already active or can be induced only in the presence of different bacterial colonizers, Hydra polyps were monocolonized with exponentially growing Curvibacter and after three days of settlement they were transferred to $1.5 \mathrm{ml}$ tubes. The polyps were subsequently exposed separately and with replication $(n=5)$ to $5000 \mathrm{CFU} / \mathrm{ml}$ to one of the five main colonizers: Curvibacter sp., Duganella sp., Undibacterium sp., Pelomonas sp. and Acidovorax sp. After $16 \mathrm{~h}$ incubation time a DNA extraction of the polyp and its surrounding water was conducted and the bacteria and phages were quantified via qPCR (see above).

Impact of phage TJ1 on microbial community. Germfree Hydra vulgaris (AEP) polyps were colonized with Duganella sp., Undibacterium sp., Acidovorax sp. and Pelomonas sp. (see above). After three days of settlement we added phage TJ1 at a concentration of $1 \times 10^{4} \mathrm{PFU} / \mathrm{ml}$ to the recolonized polyps and sterile S-Medium to polyps that served as control with replication $(n=5)$. After $24 \mathrm{~h}$ and $72 \mathrm{~h}$ we extracted DNA described above. Bacterial community composition was analyzed by amplicon sequencing of the variable region $\mathrm{V} 1-\mathrm{V} 2$ of the 16S rRNA gene using the forward primer 27F (5'-AATGATACGGCGACCACCGAGATCTACAC XXXXXXXX TATGGTAATTGT AGAGTTTGATCCTGGCTCAG-3') and reverse primer 338R (5'CAAGCAGAAGACGGCATACGAGAT XXXXXXXX AGTCAGTCAGCC TGCTGCCTCCCGTAGGAGT-3') containing the Illumina adaptor p5 (forward) and p7 (reverse) and unique MIDs (designated as XXXXXXXX). PCR reactions were performed in duplicates using Phusion Hot Start DNA Polymerase (Finnzymes, Esppoo, Finnland). PCR cycling conditions were: $98^{\circ} \mathrm{C}$ for $30 \mathrm{~s}, 30 \times\left[98^{\circ} \mathrm{C}-9 \mathrm{~s}\right.$, $55^{\circ} \mathrm{C}-30 \mathrm{~s}, 72^{\circ} \mathrm{C}-90 \mathrm{~s}$, $72^{\circ} \mathrm{C}-10 \mathrm{~min}$. PCR products were combined and purified by MinELute Gel Extraction Kit (Qiagen) after agarose gel electrophoresis. Sequencing was performed on the Illumina MiSeq platform at the sequencing facility of the Kiel Institute for Clinical Molecular Biology (IKMB). Sequence data were analysed using the MOTHUR packages(Schloss et al., 2009) according to the MISeq 
SOP (Kozich et al., 2013). In Brief, MiSeq paired-end reads were assembled and quality controlled finally resulting in 9837 sequences per sample. Sequences were grouped into operational taxonomic units (OTU) using a $97 \%$ similarity threshold. Sequences were aligned to SILVA 128 Database and taxonomically classified by RDP classifier.

Statistical data analyzes. All statistical analyzes were conducted using the statistic program $R \circledR$ (version 3.2.3 (2015-12-10) "Wooden Christmas-Tree" (Copyright (C) 2015 The R Foundation for Statistical)). The Levene's test was used to check for homogeneity of variance. Some data did not fulfill the criteria of normality. Therefore, the effect of environmental stressors on phage induction was analyzed by using a Wilcoxon-Rank-Test. The amount of phages over time and the effect of other colonizers on prophage induction were analyzed by using a generalized linear model (GLM), with a following Tukey HSD post-hoc test for multiple comparisons or pairwise comparisons. The statistical analysis of the cross-infection in vivo was conducted by ANOVA, with a following Tukey HSD post-hoc test for multiple comparisons.

\section{Results and Discussion}

Curvibacter sp. (strain AEP1.3) harbours a prophage that can be induced in culture and on Hydra. Bio-computational analysis of the genome of Curvibacter sp. (Pietschke et al., 2017) (strain AEP1.3) predicted an intact prophage within the region 444 644-484 $825 \mathrm{bp}$ and an incomplete prophage within the region (547656-566074). To test the induction capacity and to visualize the Curvibacter phage TJ1, we first induced the phage with Mitomycin C. Low concentrations of Mitomycin C between 0.05 and $0.1 \mu \mathrm{g} / \mathrm{ml}$ were sufficient to reduce the growth of the bacterial host Curvibacter (Fig. 1A). The decline in growth is an indicator for phage induction and replication. We verified the presence of phage TJ1 by gradient ultracentrifugation, negative staining with uranyl acetate and visualization via transmission electron microscopy (TEM) (Fig. 1B). Transmission electron micrographs displayed morphological similarity of phage TJ1 to Myoviridae featuring an isometric head of 50 $\mathrm{nm}$ in diameter and a contractile tail of 80 by $20 \mathrm{~nm}$ with small tail fibers (Fig. 1B).

Sequencing the linear double-stranded DNA of phage TJ1 (Supplementary Figure S1) revealed that the $37079 \mathrm{bp}$ long phage genome featured 54 proteins including capsid protein, phage tail, tail sheath and tail fiber proteins as well as transposase and lytic murein transglycosylase (Table S1). The second prophage seems to be inactive as we did not observe different morphotypes by TEM or detected it in our sequencing data. A comparison of phage TJ1 to other known phages indicated highest similarity to Burkholderia phage KS10 with 25 of proteins in common (Fig. 1C). Similarity searches of phage TJ1 by BLASTP revealed the presence of TJ1 predicted proteins in several other bacterial strains including Betaproteobacteria but also Gammaproteobacteria. Screening the genomes of these bacteria by PHASTER we could reveal that these proteins were located within prophages. Phylogenetic analysis of phage sequences by VICTOR using the GENOME-BLAST Distance Phylogenetic method (GBDP) demonstrated the presence of phylogenetic similar phages in distantly related bacteria implicating a brought host range of these phages infecting Betaproteobacteria as well as Gammaproteobacteria (Supplementary Fig. S2). 


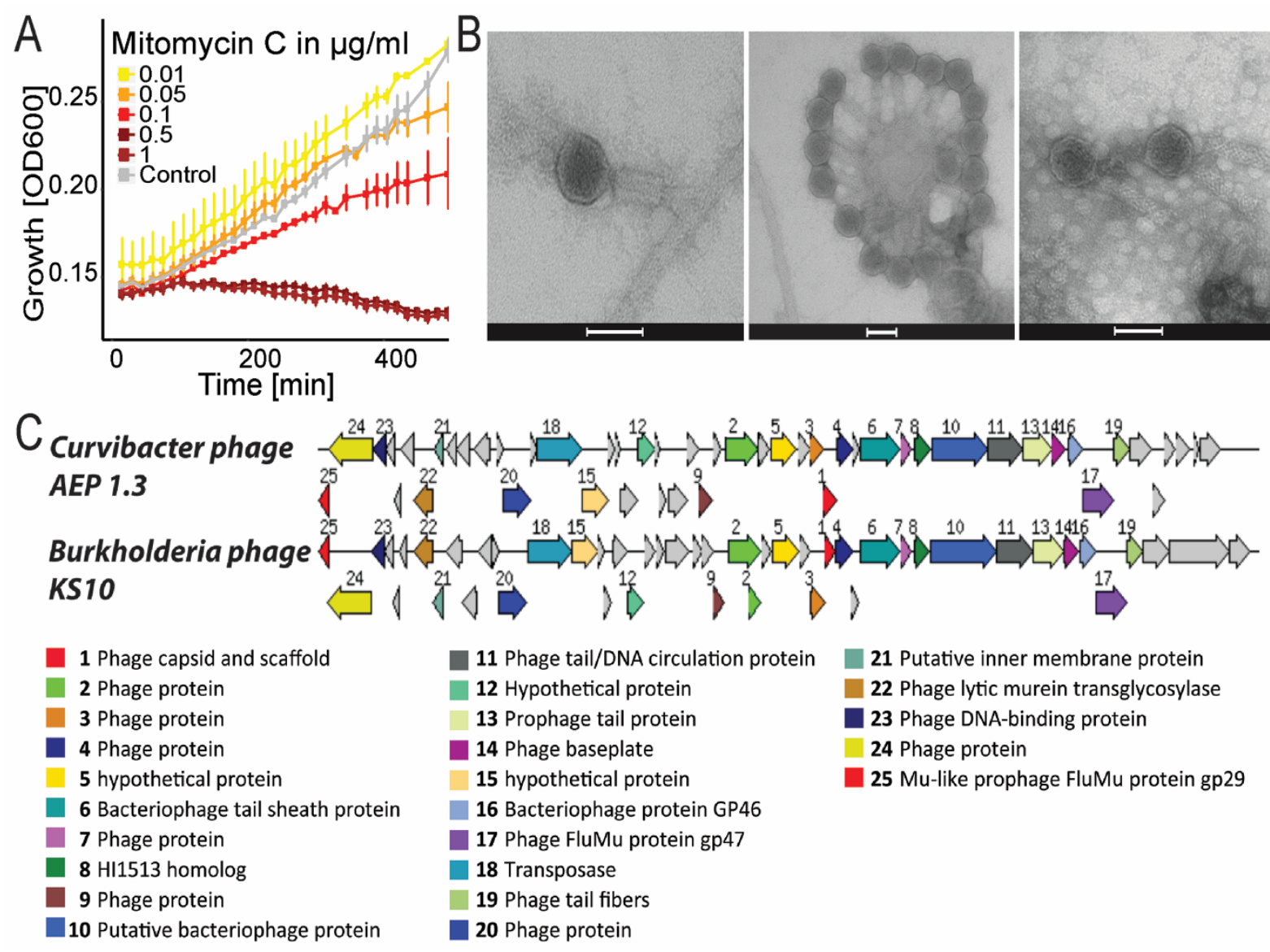

Fig 1. A: Mean growth (OD600) ( \pm SE) of Curvibacter sp. without treatment (Control, grey) and when exposed to 5 different concentrations of Mitomycin C $(n=3)$. B: Transmission Electron micrographs of Curvibacter phage TJ1 negative stained with $2 \%(\mathrm{w} / \mathrm{v})$ aqueous uranyl acetate. Scale bars represent $50 \mathrm{~nm}$. C: Annotation overview of phage TJ1 compared to Burkholderia phage KS10 (NCBI: Reference Sequence: NC_011216.1). The graphic is centered on the phage capsid coding region colored in red and numbered with 1 . Sets of genes with similar sequence are grouped with the same number and color.

To test if the prophage of Curvibacter sp. can be excised from the host genome under more natural conditions we tested the impact of different environmental factors including temperature, $\mathrm{pH}$ and nutrition on prophage induction both in culture (in vitro) (Fig. 2A) and in association with Hydra (in vivo) (Fig. 2B). We quantified phages and Curvibacter via qPCR (Fig. S3, Fig. S4) and calculated phage/Curvibacter ratio to exclude the effect of different colonization successes which highly influenced by the polyp size. When Curvibacter sp. was grown in bacterial growth media in the absence of the eukaryotic host, environmental stressors, like altered temperature, higher $\mathrm{pH}$ or higher nutrition had no significant effect on prophage induction in comparison to the control. Phage TJ1 could only be induced by Mitomycin C (Wilcoxon-Rank-Sum-test: $\mathrm{W}=0, \mathrm{p}=0.007$ ) (Fig. 2C). Living in association with Hydra phage TJ1 also be induced by Mitomycin $C$ (Wilcoxon-Rank-Sum-test: $W=0$, $p=0.01193$ (Fig. 2D). The amount of phage TJ1 produced under Mitomycin C induction was significantly higher when its bacterial host Curvibacter was living in association with the Hydra epithelium compared to the in vitro studies (One-WayANOVA, $F=10.13 p=0.002$ ). Interestingly a temperature reduction from $18^{\circ} \mathrm{C}$ to $12^{\circ} \mathrm{C}$ affected the number of induced phages only if the bacterial host Curvibacter sp. lived in associated with Hydra (Wilcoxon-Rank-Sum-test: $W=25, p=0.01193$ ). Changes in $\mathrm{pH}$-values, elevated temperature or nutrition did not lead to a significant prophage 
induction, neither in vitro, nor in vivo (Fig. 2B, 2D). This observation suggests that lysogenesis is maintained and phages are not induced under a natural range of variable environmental condition. As phage TJ1 was not detectable in the virome data set of Hydra (Grasis et al., 2014), ambient or elevated water temperature also seems to have no effect on prophage induction on polyps, that are associated with a complex, natural bacterial community.

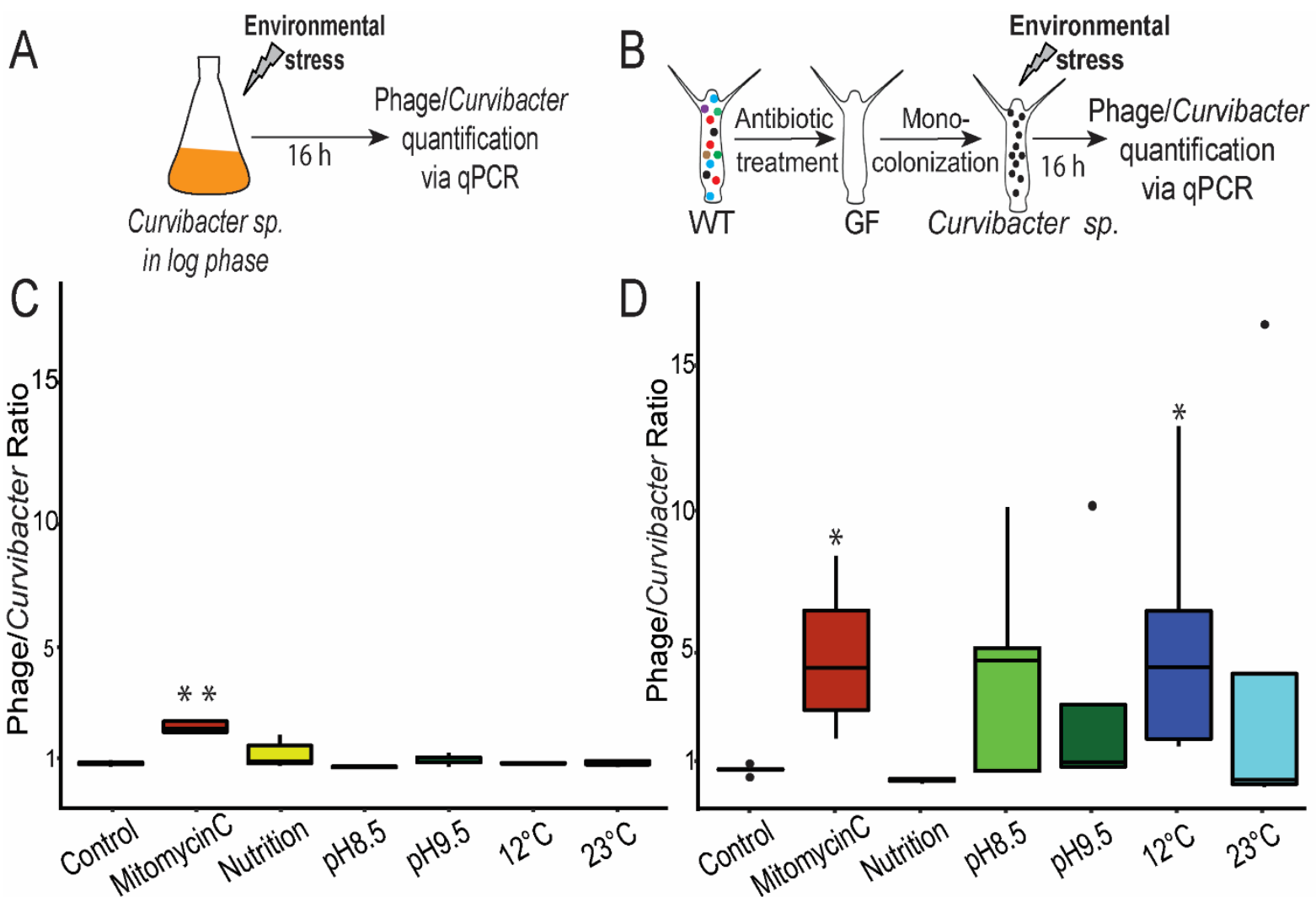

Fig 2. A: In vitro experimental set-up. Curvibacter sp. were grown at log phase and exposed to several environmental stressors. Prophage induction was quantified via qPCR $(n=5)$. B: In vivo experimental set-up. WT polyps were treated with antibiotics to generate germfree Hydra. These germfree polyps were recolonized in mono-association with Curvibacter and exposed to different environmental stressors. Prophage induction was quantified via qPCR $(n=5)$; C: Boxplot of phage/Curvibacter ratio after exposure to the environmental stressors. Stars $\left({ }^{*}=p<0.05,{ }^{* *}=p<0.01\right)$ indicate significantly differences between treatment and control (Wilcoxon rank sum test). D: Boxplot of phage/Curvibacter ratios after the polyps were exposed to the environmental stressors. Stars $\left({ }^{*}=p<0.05,{ }^{* *}=p<0.01\right)$ indicate significantly differences between treatment and control (Wilcoxon rank sum test).

The observation that the prophage can be induced in vivo by lowering the water temperature is interesting since temperature serves as an important environmental cue in Hydra and can affect many developmental processes including body size and budding (Bode et al., 1973; Bisbee, 1973; Stiven, 1965) as well as onset of sexual reproduction (Littlefield et al., 1991).

Prophage excision is expected to be strongest when bacteria are exponentially growing (Madera et al., 2009), due to the high expression of genes involved in the SOS response of the bacterium (Nanda et al., 2014). In the glycocalyx of Hydra epithelia, we would expect decreased bacterial growth compared to liquid culture conditions due to nutrient limitation and thereof a reduction of phage inducibility. However, we observed the opposite; phage inducibility was significantly higher (OneWay-ANOVA, $F=10.13 p=0.002$ ) when bacteria were associated with their host. The 
observed differences in the induction capacity add support to the view that the host environment has a strong influence on phage bacteria interactions (De Paepe et al., 2016). Important factors in the epithelial-derived mucus layer may include nutrients as well as components of the innate immune system (Deines et al., 2017; Pietschke et al., 2017). However, there are also other factors that might play a role in the differential induction capacity. Firstly, we cannot exclude a possible additive effect of residual antibiotics on prophage induction which were used in the process of generating germfree polyps, although we stopped antibiotic treatment 6 days before the experiments and washed polyps twice in sterile Hydra medium before they were used for all experiments. Secondly, dilution or density dependent issues may lead to a lower induction rate in vitro compared to in vivo conditions. Recently we predicted by mathematical modelling that at low frequencies the main rout of phage TJ1 production is via the lytic pathway, while at high densities the lysogenic cycle is favored (Li et al., 2017).

Curvibacter phage TJ1 can infect a broad range of Hydra associated bacteria. To test the ability of phage TJ1 to cross-infect other bacteria we spotted purified phages onto a lawn of Hydra associated bacteria and used plaque formation as sign of infection success. For a variety of bacteria belonging to Betaproteobacteria we could observe plaque formation, while no plaque formation was visible for Gammaproteobacteria and Bacteroidetes (Supplementary Table S2). Phage TJ1 could not only infect closely related bacterial strains affiliating to the same family as their original bacterial host (Comamonadaceae) but seems to be lytic in a broad spectrum of bacteria. This includes Undibacterium sp. and Duganella sp., (Oxalobacteraceae), as well as Vogesella sp., a less abundant bacterium of Hydra, belonging to the order Neisseriales (Supplementary Table S2). To examine whether phage TJ1 can cross-infect bacteria not only in vitro but also when they are living on Hydra, we monocolonized germfree Hydra with the most abundant bacterial colonizer: Curvibacter sp., Duganella sp., Undibacterium sp., Acidovorax sp. and Pelomonas sp. (Fraune et al., 2014) and subsequently inoculated them with phage TJ1. After $0 \mathrm{~h}, 24 \mathrm{~h}$ and $72 \mathrm{~h}$ post phage infection we quantified living bacterial cells by counting (CFU/polyp) and estimated phage/bacteria ratio by qPCR (Fig. 3A). While the phage did not interfere with the growth of Curvibacter sp. and Acidovorax sp., we observed a significant reduction of Duganella sp. and Undibacterium sp. after $72 \mathrm{~h}$ post phage exposure (One-way ANOVA, Duganella: $F=4.111$; $p<0.006$, Undibacterium: $F=3.973 ; p=0.013$ ) (Fig. 3B). Interestingly we observed a significant reduction of CFUs in polyps that were monocolonized with Pelomonas sp. $72 \mathrm{~h}$ post phage infection (One-way ANOVA, $F=22.92, p<0.001$ ) (Fig. 3B), although Pelomonas sp. could not be infected by phage TJ1 in in vitro experiments (Supplementary Table 1). The impact of phage TJ1 on bacterial numbers (Fig. 3B) was accompanied by an elevated amount of phages (Fig. 3C). The number of phages increased significantly over time in polyps which were recolonized with Undibacterium ( $G L M=2.7373, p=0.0103)$, Duganella $(G L M, F=2.7373, p=0.0246$ ) and Pelomonas (GLM, F = 2.7373, 0-24h: $p=0.0011 ; 0-72 h: p<0.0024)$ ) (Fig. 3C). This implies a successful infection and replication of phage TJ1. There was no significant increase of phages detectable in polyps recolonized with Curvibacter sp. or Acidovorax sp. (Fig. 3C). 

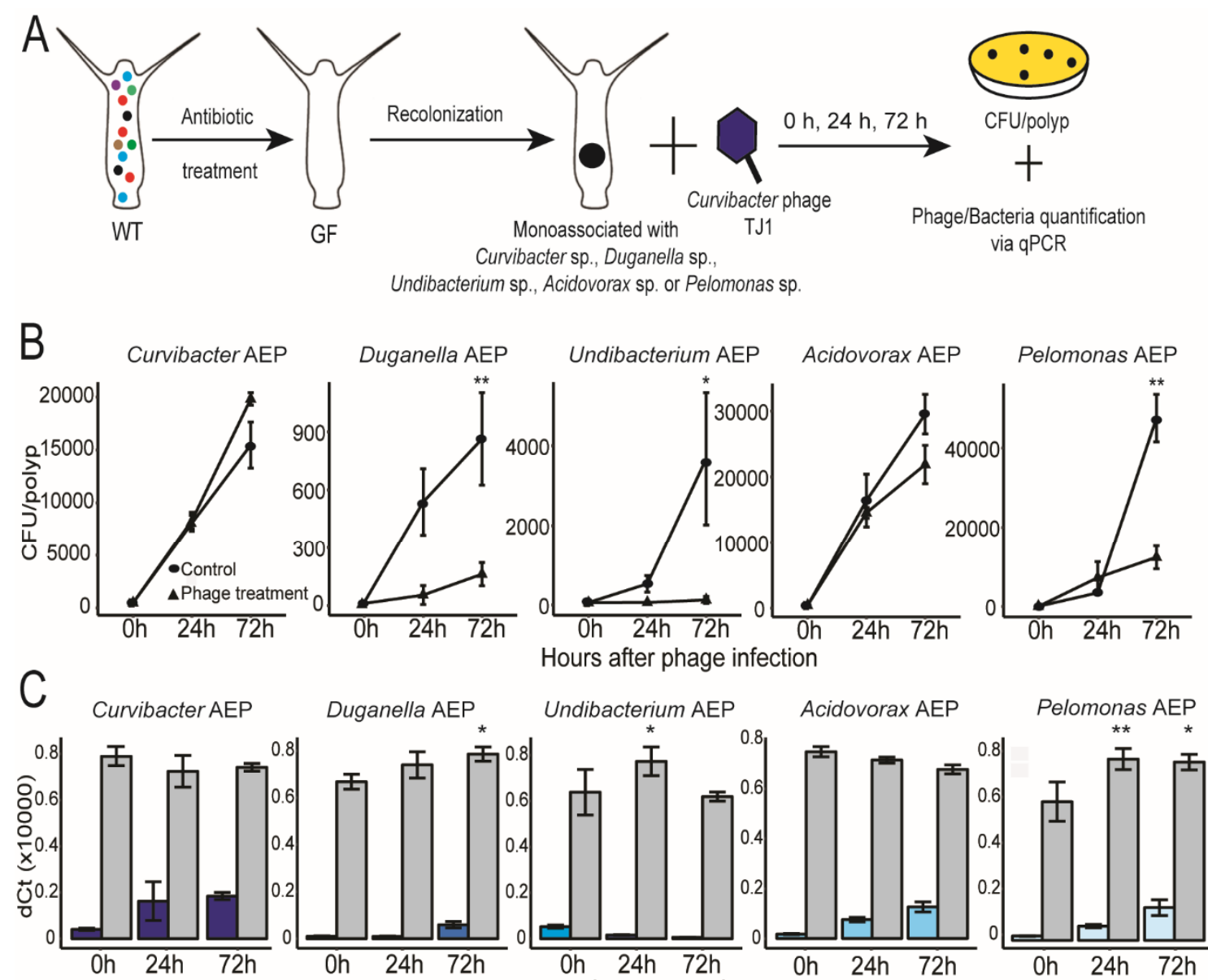

Undibacterium AEP Acidovorax AEP

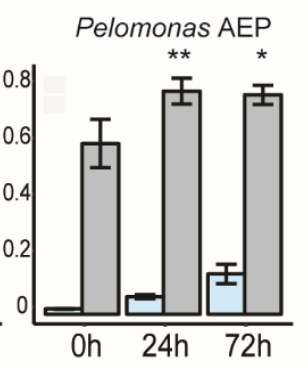

Hours after phage infection

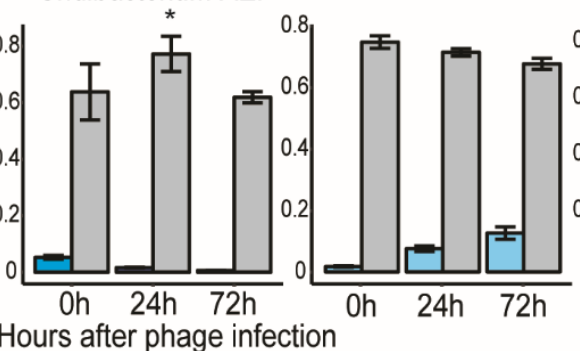

Phage TJ1

Bacteria

Fig 3. A: Experimental set-up. WT polyps were treated with antibiotics to generate germfree Hydra. These germfree polyps were recolonized in mono-association with one of the 5 main colonizer and either treated with phage TJ1 or with SM-Buffer as a control. The bacteria were quantified via plating and counting the CFU/polyp and the phages were quantified via qPCR. B: Mean CFU/polyp ( \pm SE) over time of Curvibacter sp., Duganella sp., Undibacterium sp., Acidovorax sp. or Pelomonas sp. on Hydra with phages $(\Delta)$ or without $(\bullet)(n=5)$. Stars $\left({ }^{*}=p<0.05,{ }^{* *}=p<0.01\right)$ indicate significantly difference between phage treatment and control (One-way-ANOVA). C: Bar plot of deltaCt values of Curvibacter (blue) and phage (grey) over time in the phage TJ1 treated polyps monocolonized with Curvibacter sp., Duganella sp., Undibacterium sp., Acidovorax sp. or Pelomonas sp. $(n=5)$. Stars $\left({ }^{*}=p<0.05,{ }^{* *}=p<0.01\right)$ indicate significantly difference between the time of incubation in comparison to $0 \mathrm{~h}(\mathrm{GLM})$.

Similar broad host ranges (polyvalency) have been described for other phages that belong to the Myoviridae (Goodridge et al., 2003) and other freshwater phages (Malki et al., 2015). Polyvalency has been suggested to be linked to poor-nutrient conditions as an adaptation to low host cell numbers in aquatic environments (Chibani-Chennoufi et al., 2004) and is also wide spread in bacteriophages that infect Burkholderiales (Langley et al., 2003; Kawasaki et al., 2009). In this context the Burkholderia cepacia complex (BCC) group of bacteria is intensively studied. This group of bacteria includes beneficial and pathogenic bacteria of plants but also human opportunistic pathogens, such as Burkholderia cenocepacia causing pulmonary infection in the background of cystic fibrosis and chronic granulomatous disease (Mahenthiralingam et al., 2005). Several phages have been characterized for their ability to control B. cenocepacia among those Burkholderia phage KS10 (Goudie et al., 2008), which shared 25 of 54 proteins with phage TJ1. The presence 
of phylogenetic similar prophages in distantly related bacteria Betaproteobacteria as well as Gammaproteobacteria (Supplementary Fig. S2) supports our findings of a brought host range.

Our data provide direct evidence that phage TJ1 can affect the abundance of symbiotic bacteria which are stably associated with their eukaryotic host. Due to its ability to infect and kill other Curvibacter strains from different Hydra species, phage TJ1 may function as a weapon against these bacteria and secure its host the dominant position on the glycocalyx of Hydra vulgaris (AEP).

Curvibacter can use phage TJ1 to shape the Hydra associated microbiota. Given the above results, phage TJ1 may contribute to the regulation of the microbial composition. However, to exert its regulatory function phage TJ1 must switch from a lysogenic to a lytic life cycle even under normal environmental conditions. According to our recently published modelling approach the lytic pathway of phage TJ1 is predicted to be favored at low frequencies of Curvibacter sp. and high frequencies of Duganella sp. (Li et al., 2017). For this we thought that the presence of different bacterial strains is sufficient to exert the regulatory function of phage TJ1. To test this, we monocolonized Hydra vulgaris (strain AEP) with Curvibacter sp. and exposed them separately to five bacterial strains commonly found on Hydra vulgaris (strain AEP): Curvibacter sp. (double recolonization as control), Duganella sp., Undibacterium sp., Acidovorax sp. and Pelomonas sp.. After $16 \mathrm{~h}$ we quantified phages, Curvibacter (Fig. S4) and all bacteria (Fig. S5) by qPCR (Fig. 4A). Intriguingly, we observed a significant higher phage/Curvibacter ratio compared to the control treatment, when Curvibacter mono-associated polyps were exposed to phage TJ1 sensitive bacteria including Duganella (Generalized linear model (GLM), $\mathrm{F}=6.9518, \mathrm{p}=0.03723$ ), Pelomonas (GLM, $\mathrm{F}=6.9518, \mathrm{p}=0.02432$ ) and Undibacterium (GLM, $F=6.9518, p=0.00299$ ) (Fig. 4B).

The addition of bacterial strains that were resistant to phage infection, such as Curvibacter sp. and Acidovorax sp., did not lead to significant changes in the ratio between phage and Curvibacter sp. (Fig. 4B). An elevated prophage induction in Curvibacter sp. or cross-infectivity of these bacterial strains therefore can be excluded. The observation that phage/Curvibacter ratios increased in the presence of specific bacteria clearly demonstrates that the prophage of Curvibacter can exert a regulatory function on the microbiota composition. 

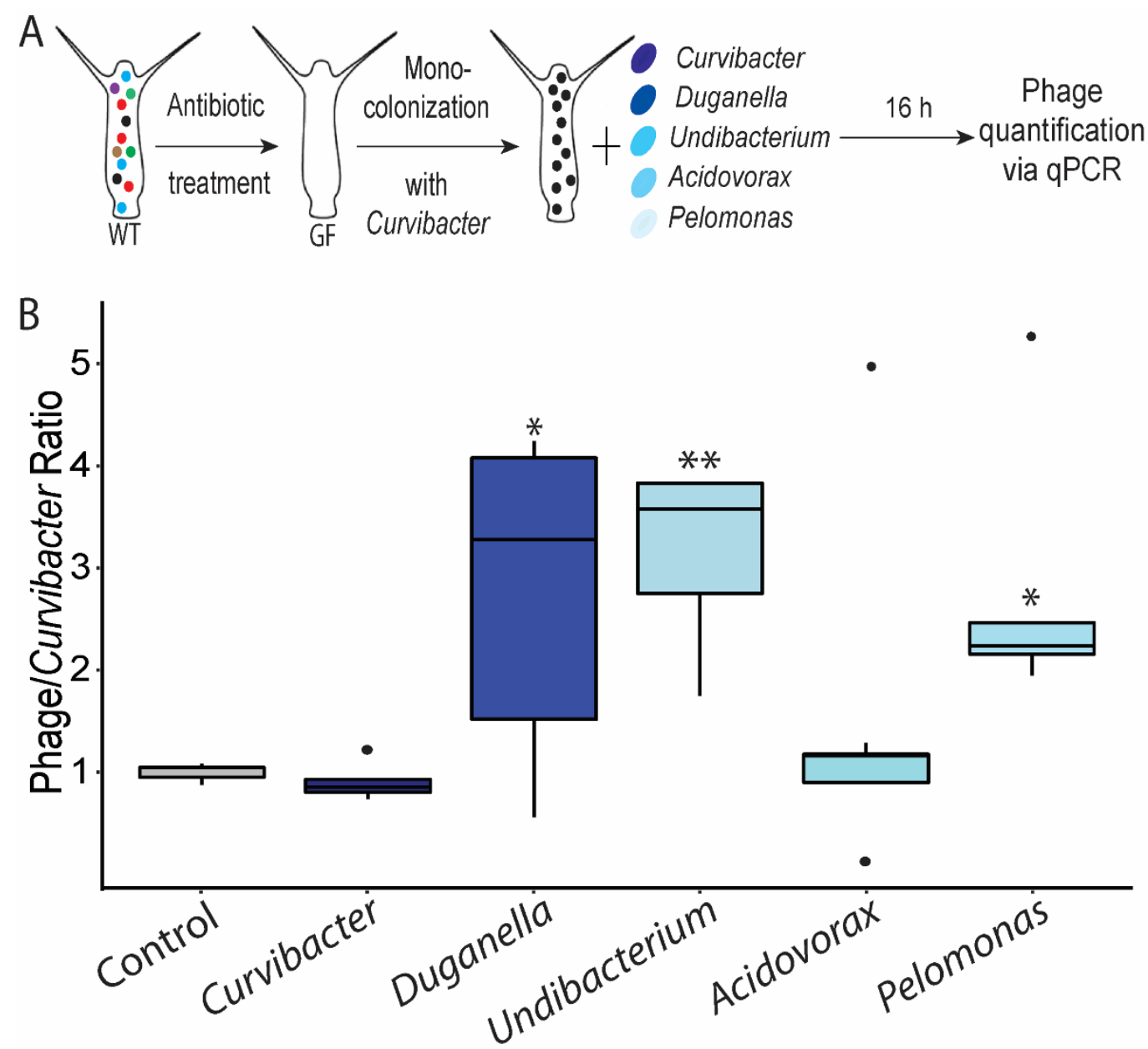

Fig 4. A: Experimental set-up; WT polyps were treated with antibiotics to generate germfree Hydra. These germfree polyps were recolonized in mono-association with Curvibacter and exposed to one of Hydras main colonizer (Curvibacter, Duganella, Undibacterium, Acidovorax or Pelomonas) or sterile Hydra culture medium as control. Phages were quantified via qPCR $(n=5)$; B: Boxplot of phage/Curvibacter ratio after the polyps were exposed to the bacteria. Stars $\left({ }^{*}=p<0.05,{ }^{* *}=p<0.01\right)$ indicate significantly differences between treatment and control (Generalized linear model).

We could show that already the addition of bacterial strains to the surrounding of monocolonized Hydra leads to phage replication. This might be triggered by small signal molecules released by bacteria (Ghosh et al., 2009) or the ability of low abundant phages to cross-infect new colonizing bacteria. It could also be speculated that an increased abundance of bacteria stimulate the immune response of the eukaryotic host Hydra (Franzenburg et al., 2012), which may lead to prophage induction. Nevertheless, this experiment clearly shows that phage TJ1 is active on Hydra without any additional artificial stimulation and interferes with microbial colonization.

Impact of Curvibacter phage TJ1 on microbial community composition on Hydra. To validate the impact of phage TJ1 on a bacterial community level, we exposed polyps that were recolonized with Duganella, Undibacterium, Acidovorax and Pelomonas to phage TJ1. After $24 \mathrm{~h}$ and $72 \mathrm{~h}$ we analyzed the bacterial community composition by $16 \mathrm{~S}$ rRNA amplicon sequencing and observed a reduction in the relative abundance of Duganella (GLM, $F=14.077,72 \mathrm{~h}: \mathrm{p}=0.0233$ ) and Pelomonas (GLM, $F=14.077,24 \mathrm{~h}: \mathrm{p}=0.0064,72 \mathrm{~h}: \mathrm{p}<0.001$ ) in the phage treatments compared to the control (Fig 5A). This reduction was accompanied by a 
relative increase of the phage resistant bacterium Acidovorax (GLM, F=14.077, $24 \mathrm{~h}$ : $\mathrm{p}<0.001,72 \mathrm{~h}: \mathrm{p}<0.001$ ) (Fig. $5 \mathrm{~B}$ ). The recolonization of Undibacterium was not successful and therefor absent in treatment and control. However, our experiment demonstrates for a bacterial community with reduced complexity that the presence of a temperate phage has the potential to cause tremendous shifts in the bacterial community composition. Considering that all host associated bacterial communities also feature a diverse phage community e.g. the human gut (Manrique et al., 2016) complex phage regulated processes of the bacterial community can be expected. Our results demonstrate that already the work with bacterial isolates should consider that single bacterial strains can be associated with prophages. Environmental and bacterial frequency depend factors that affect lysogenic or lytic decision of phages can impair experimental outcomes. Moreover, cross-infection and horizontal gene transfer are additional factors that should be taken into account when studying bacteria-bacterial as well as bacteria-host interactions.
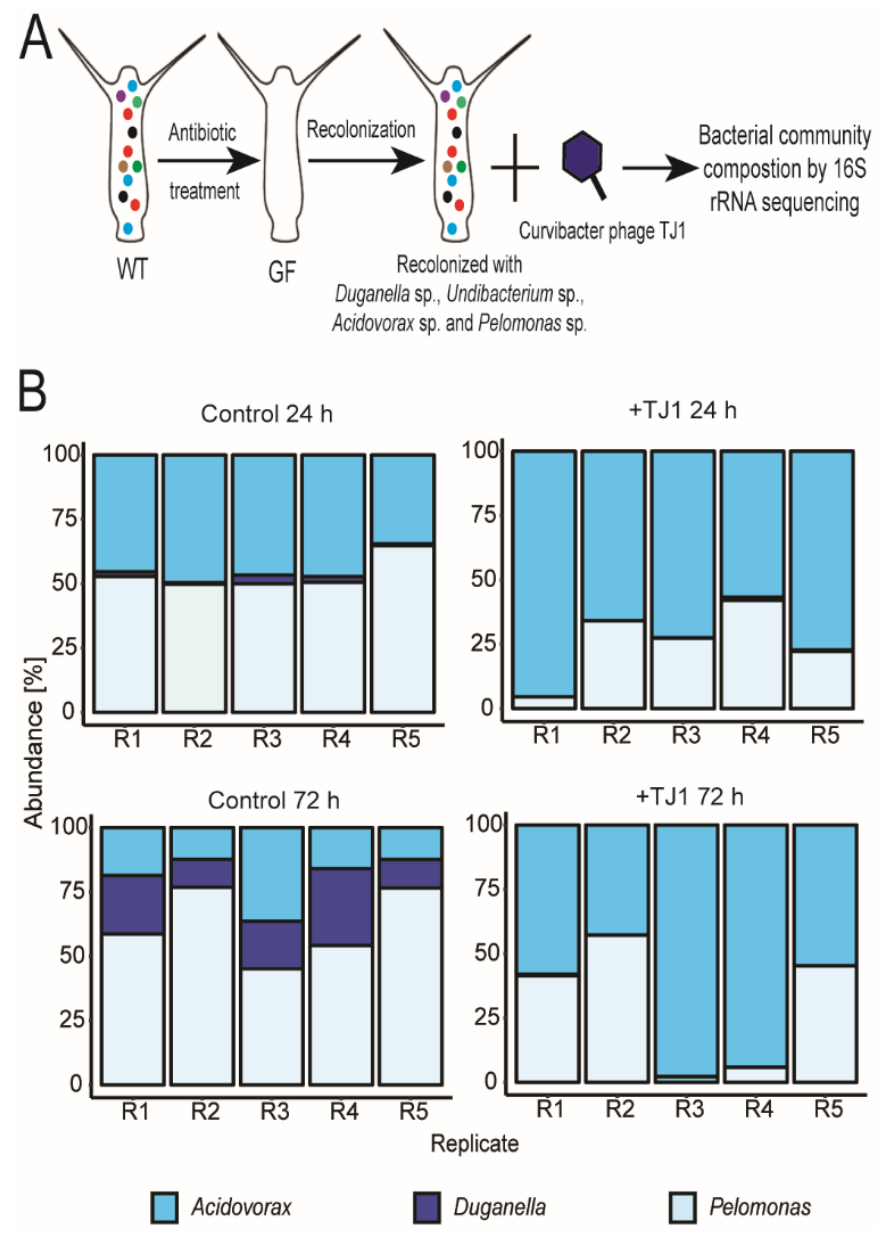

Fig 5 A: Experimental set-up; WT polyps were treated with antibiotics to generate germfree $H y d r a$. Germfree polyps were recolonized with Duganella, Undibacterium, Acidovorax and Pelomonas. Recolonised polyps were exposed to Curvibacter phage TJ1. After $24 \mathrm{~h}$ and $72 \mathrm{~h}$ hours bacterial community composition was analysed by $16 \mathrm{~S}$ rRNA amplicon sequencing $(n=5)$. As control served recolonized polyps that were exposed to Hydra medium; B: Barplots illustrate the relative abundance of Duganella sp., Undibacterium sp., Acidovorax sp. and Pelomonas sp. of controls and phage treated polyps after $24 \mathrm{~h}$ and $72 \mathrm{~h}$. 


\section{Conclusion}

This study elucidates in the early emerging metazoan Hydra the potential regulatory role of prophages and uncovers the capability of Curvibacter sp. phage TJ1 to directly affect and shape the Hydra associated microbiota. Curvibacter sp., the most dominant colonizer of Hydra vulgaris (AEP), is associated with a prophage which can be induced and cross-infect different bacterial strains. Observed differences in both, prophage excision from the host genome and infectivity of phage TJ1 between experiments in culture and on Hydra imply that the habitat of the prokaryotic host and/or bacterial frequency dependent factors influence (bacterial) host-phage interactions. In conclusion, prophages are hidden parts of the microbiome and can interfere with bacteria-bacteria interactions. Therefore prophages have the potential to influence the composition of host associated bacterial communities.

\section{Acknowledgements}

The work was supported by grants from the Deutsche Forschungsgemeinschaft (DFG), the Collaborative Research Center 1182 ("Origin and Function of Metaorganisms" subproject A4 to SF and TB). TL gratefully appreciates support from the Volkswagen Foundation ("Experiment!- In search of bold research ideas" $A z$ 91135). TB acknowledge support from the Canadian Institute for Advanced Research (CIFAR).

\section{Competing Interests}

The authors declare no competing financial interests

\section{References}

Adams M. (1959). Assay of phage by agar layer method. Bacteriophages Intersci Publ Inc, New York, NY, 450-454.

Altschul SF, Madden TL, Schäffer AA, Zhang J, Zhang Z, Miller W, et al. (1997). Gapped BLAST and PSI-BLAST: A new generation of protein database search programs. Nucleic Acids Res. e-pub ahead of print, doi: 10.1093/nar/25.17.3389. Arndt D, Grant JR, Marcu A, Sajed T, Pon A, Liang Y, et al. (2016). PHASTER: a better, faster version of the PHAST phage search tool. Nucleic Acids Res 44: W16W21.

Bairoch A, Apweiler R. (2000). The SWISS-PROT protein sequence database and its supplement TrEMBL in 2000. Nucleic Acids Res. e-pub ahead of print, doi: 10.1093/nar/28.1.45.

Bankevich A, Nurk S, Antipov D, Gurevich AA, Dvorkin M, Kulikov AS, et al. (2012). SPAdes: a new genome assembly algorithm and its applications to single-cell sequencing. J Comput Biol 19: 455-77.

Bates KA, Clare FC, O'Hanlon S, Bosch J, Brookes L, Hopkins K, et al. (2018). Amphibian chytridiomycosis outbreak dynamics are linked with host skin bacterial community structure. Nat Commun 9: 693.

Bisbee BJW. (1973). Size determination in Hydra: The roles of growth and budding. J Embryol Exp Morphol 30: 1-19.

Bode H, Berking S, David CN, Gierer A, Schaller H, Trenkner E. (1973). Quantitative analysis of cell types during growth and morphogenesis in Hydra. Wilhelm Roux Arch für Entwicklungsmechanik der Org 171: 269-285.

Bohannan BJM, Lenski RE. (2000). Linking genetic change to community evolution: Insights from studies of bacteria and bacteriophage. Ecol Lett 3: 362-377.

Bolger AM, Lohse M, Usadel B. (2014). Trimmomatic: a flexible trimmer for Illumina 
sequence data. Bioinformatics 30: 2114-20.

Borodovsky M, Mclninch J. (1993). GENMARK: Parallel gene recognition for both DNA strands. Comput Chem. e-pub ahead of print, doi: 10.1016/0097-

8485(93)85004-V.

Bosch TC, Krylow SM, Bode HR, Steele RE. (1988). Thermotolerance and synthesis of heat shock proteins: these responses are present in Hydra attenuata but absent in Hydra oligactis. Proc Natl Acad Sci U S A 85: 7927-31.

Bosch TCG, McFall-Ngai MJ. (2011). Metaorganisms as the new frontier. Zoology 114: 185-190.

Bossi L, Fuentes J a, Mora G, Figueroa-bossi N. (2003). Prophage Contribution to Bacterial Population Dynamics Prophage Contribution to Bacterial Population Dynamics. J Bacteriol 185: 6467-6471.

Burns N, James CE, Harrison E. (2015). Polylysogeny magnifies competitiveness of a bacterial pathogen in vivo. Evol Appl 8: 346-351.

Chibani-Chennoufi S, Bruttin A, Dillmann ML, Brüssow H. (2004). Phage-host interaction: An ecological perspective. J Bacteriol 186: 3677-3686.

Deines P, Lachnit T, Bosch TCG. (2017). Competing forces maintain the Hydra metaorganism. Immunol Rev 279: 123-136.

El-Gebali S, Mistry J, Bateman A, Eddy SR, Luciani A, Potter SC, et al. (2019). The Pfam protein families database in 2019. Nucleic Acids Res. e-pub ahead of print, doi: 10.1093/nar/gky995.

Franzenburg S, Fraune S, Altrock PM, Künzel S, Baines JF, Traulsen A, et al. (2013a). Bacterial colonization of Hydra hatchlings follows a robust temporal pattern. ISME J 7: 781-90.

Franzenburg S, Fraune S, Kunzel S, Baines JF, Domazet-Loso T, Bosch TCG. (2012). MyD88-deficient Hydra reveal an ancient function of TLR signaling in sensing bacterial colonizers. Proc Natl Acad Sci. e-pub ahead of print, doi:

10.1073/pnas.1213110109.

Franzenburg S, Walter J, Künzel S, Wang J, Baines JF, Bosch TCG, et al. (2013b). Distinct antimicrobial peptide expression determines host species-specific bacterial associations. Proc Natl Acad Sci U S A 110: E3730-8.

Fraune S, Anton-Erxleben F, Augustin R, Franzenburg S, Knop M, Schröder K, et al. (2014). Bacteria-bacteria interactions within the microbiota of the ancestral metazoan Hydra contribute to fungal resistance. ISME J. e-pub ahead of print, doi:

10.1038/ismej.2014.239.

Ghosh D, Roy K, Williamson KE, Srinivasiah S, Wommack KE, Radosevich M. (2009). Acyl-homoserine lactones can induce virus production in lysogenic bacteria: An alternative paradigm for prophage induction. Appl Environ Microbiol 75: 71427152.

Gómez P, Buckling A. (2011). Bacteria-phage antagonistic coevolution in soil. Science (80- ) 332: 106-109.

Goodrich JK, Waters JL, Poole AC, Sutter JL, Koren O, Blekhman R, et al. (2014). Human genetics shape the gut microbiome. Cell 159: 789-799.

Goodridge L, Gallaccio A, Mansel W, Griffiths MW. (2003). Morphological , Host Range, and Genetic Characterization of Two Coliphages Morphological , Host Range, and Genetic Characterization of Two Coliphages. Appl Environ Microbiol 69: 5364-5371.

Goudie AD, Lynch KH, Seed KD, Stothard P, Shrivastava S, Wishart DS, et al. (2008). Genomic sequence and activity of KS10, a transposable phage of the Burkholderia cepacia complex. BMC Genomics 9. e-pub ahead of print, doi: 10.1186/1471-2164-9-615. 
Grasis JA, Lachnit T, Anton-Erxleben F, Lim YW, Schmieder R, Fraune S, et al. (2014). Species-specific viromes in the ancestral holobiont hydra. PLoS One 9. e-pub ahead of print, doi: 10.1371/journal.pone.0109952.

Hemmrich G, Anokhin B, Zacharias H, Bosch TCG. (2007). Molecular phylogenetics in Hydra, a classical model in evolutionary developmental biology. Mol Phylogenet Evol 44: 281-290.

Imamovic L, Ballesté E, Jofre J, Muniesa M. (2010). Quantification of Shiga toxinconverting bacteriophages in wastewater and in fecal samples by real-time quantitative PCR. Appl Environ Microbiol 76: 5693-5701.

Kawasaki T, Shimizu M, Satsuma H, Fujiwara A, Fujie M, Usami S, et al. (2009). Genomic characterization of Ralstonia solanacearum phage $\varphi R S B 1$, a T7-like widehost-range phage. J Bacteriol 91: 422-427.

Koskella B, Meaden S. (2013). Understanding bacteriophage specificity in natural microbial communities. Viruses 5: 806-823.

Kozich JJ, Westcott SL, Baxter NT, Highlander SK, Schloss PD. (2013).

Development of a dual-index sequencing strategy and curation pipeline for analyzing amplicon sequence data on the miseq illumina sequencing platform. Appl Environ Microbiol 79: 5112-5120.

Langley R, Kenna DT, Vandamme P, Ure R, Govan JRW. (2003). Lysogeny and bacteriophage host range within the Burkholderia cepacia complex. J Med Microbiol 52: 483-490.

Lenski RE. (1988). Dynamics of Interactions between Bacteria and Virulent Bacteriophage. In: Marshall KC (ed). Advances in Microbial Ecology. Springer US: Boston, MA, pp 1-44.

Li X-Y, Lachnit T, Fraune S, Bosch TCG, Traulsen A, Sieber M. (2017). Temperate phages as self-replicating weapons in bacterial competition. J R Soc Interface 14: 20170563.

Li X-Y, Pietschke C, Fraune S, Altrock PM, Bosch TCG, Traulsen A. (2015). Which games are growing bacterial populations playing? J R Soc Interface 12: 20150121. Littlefield CL, Finkemeier C, Bode HR. (1991). Spermatogenesis in Hydra oligactis. Dev Biol 146: 292-300.

Livny J, Friedman DI. (2004). Characterizing spontaneous induction of Stx encoding phages using a selectable reporter system. Mol Microbiol 51: 1691-1704.

Madera C, García P, Rodríguez A, Suárez JE, Martínez B. (2009). Prophage induction in Lactococcus lactis by the bacteriocin Lactococcin 972. Int J Food Microbiol 129: 99-102.

Mahenthiralingam E, Urban TA, Goldberg JB. (2005). The multifarious, multireplicon Burkholderia cepacia complex. Nat Rev Microbiol 3: 144-156.

Malki K, Kula A, Bruder K, Sible E, Hatzopoulos T, Steidel S, et al. (2015).

Bacteriophages isolated from Lake Michigan demonstrate broad host-range across several bacterial phyla. Virol J 12: 1-5.

Manrique P, Bolduc B, Walk ST, Oost J Van Der, Vos WM De, Young MJ. (2016). Healthy human gut phageome. Proc Natl Acad Sci. e-pub ahead of print, doi: 10.1073/pnas.1601060113.

Meier-Kolthoff JP, Göker M. (2017). VICTOR: genome-based phylogeny and classification of prokaryotic viruses. Bioinformatics. e-pub ahead of print, doi: 10.1093/bioinformatics/btx440.

Nanda AM, Heyer A, Krämer C, Grünberger A, Kohlheyer D, Frunzke J. (2014). Analysis of SOS-induced spontaneous prophage induction in Corynebacterium glutamicum at the single-cell level. J Bacteriol 196: 180-188.

Nanda AM, Thormann K, Frunzke J. (2015). Impact of spontaneous prophage 
induction on the fitness of bacterial populations and host-microbe interactions. $J$ Bacteriol 197: 410-419.

Oakey HJ, Owens L. (2000). A new bacteriophage, VHML, isolated from a toxinproducing strain of Vibrio harveyi in tropical Australia. J Appl Microbiol 89: 702-709. De Paepe M, Leclerc M, Tinsley CR, Petit M-A. (2014). Bacteriophages: an underestimated role in human and animal health? Front Cell Infect Microbiol 4: 39. De Paepe M, Tournier L, Moncaut E, Son O, Langella P, Petit MA. (2016). Carriage of $\lambda$ Latent Virus Is Costly for Its Bacterial Host due to Frequent Reactivation in Monoxenic Mouse Intestine. PLoS Genet 12: 1-20.

Pietschke C, Treitz C, Forêt S, Schultze A, Künzel S, Tholey A, et al. (2017). Host modification of a bacterial quorum-sensing signal induces a phenotypic switch in bacterial symbionts. Proc Natl Acad Sci 201706879.

Ranquet C, Toussaint A, De Jong H, Maenhaut-Michel G, Geiselmann J. (2005). Control of bacteriophage Mu lysogenic repression. J Mol Biol 353: 186-195.

Refardt D. (2012). Real-time quantitative PCR to discriminate and quantify lambdoid bacteriophages of Escherichia coli K-12. Bacteriophage 2: 98-104.

Van Rensburg JJ, Lin H, Gao X, Toh E, Fortney KR, Ellinger S, et al. (2015). The human skin microbiome associates with the outcome of and is influenced by bacterial infection. MBio 6. e-pub ahead of print, doi: 10.1128/mBio.01315-15.

Rosenberg E, Koren O, Reshef L, Efrony R, Zilber-Rosenberg I. (2007). The role of microorganisms in coral health, disease and evolution. Nat Rev Microbiol 5: 355-362. Schloss PD, Westcott SL, Ryabin T, Hall JR, Hartmann M, Hollister EB, et al. (2009). Introducing mothur: Open-source, platform-independent, community-supported software for describing and comparing microbial communities. Appl Environ Microbiol 75: 7537-7541.

Sekiguchi M, Takagi Y. (1960). Effect of mitomycin C on the synthesis of bacterial and viral deoxyribonucleic acid. BBA - Biochim Biophys Acta 41: 434-443.

Stiven AE. (1965). Hydra pseudoligactis, Chlorohydra viridissima,.

Suttle C a. (2007). Marine viruses--major players in the global ecosystem. Nat Rev Microbiol 5: 801-12.

Thurber R V, Haynes M, Breitbart M, Wegley L, Rohwer F. (2009). Laboratory procedures to generate viral metagenomes. Nat Protoc 4: 470-483.

Wattam AR, Davis JJ, Assaf R, Boisvert S, Brettin T, Bun C, et al. (2017). Improvements to PATRIC, the all-bacterial Bioinformatics Database and Analysis Resource Center. Nucleic Acids Res 45: D535-D542.

(2019). UniProt: a worldwide hub of protein knowledge. Nucleic Acids Res. e-pub ahead of print, doi: 10.1093/nar/gky1049. 